\title{
Experimental Research on Burning Rate, Vertical Velocity and Radiation of Medium-Scale Fire Whirls
}

\author{
KUIBIN ZHOU, NAIAN LIU, and KOYU SATOH \\ State Key Laboratory of Fire Science \\ University of Science and Technology of China \\ Hefei, Anhui 230026, China
}

\begin{abstract}
Fire whirls are often reported in forest and urban fires with devastating damage to life and property. This work conducted experiments using a medium-scale facility to study the initiation mechanism, the vertical velocity and radiative heat transfer of fire whirls. Heptane was used as the fuel in the experiments. The variations of burning rates versus time indicate that the drag force on the root of the flame plays an important role in the initiation and decay of a fire whirl. Analyses show that the pressure difference due to whirling, characterized by the circulation $\Gamma$, significantly affects the vertical centerline velocity. The variation of the vertical centerline velocity is correlated by $V_{z} \sim z^{0.17} \Gamma^{0.22}$ in the flaming zone and $V_{z} \sim z^{-0.66} \Gamma^{0.77}$ in the plume zone. The radiation analysis shows that the heat flux in the vertical direction reaches a maximum at $z / H \approx 0.4$ for fire whirls, and the variation of radiative heat flux in the radial direction can be correlated by $\dot{q}^{\prime \prime}(r) \sim r^{-m}$, where $m$ is nearly 1.1 for both fire whirls and general pool fires. It is also found that the radiative fraction of fire whirls is nearly $42 \%, 1.4$ times as large as that for general pool fires. The radiative heat feedback fraction is shown to increase with pool diameter, similarly as for general pool fires.
\end{abstract}

KEYWORDS: fire whirl, radiation, centerline velocity, burning rate, pool fires.

\section{NOMENCLATURE LISTING}

$c_{p, \infty} \quad$ specific heat of ambient air at atmospheric pressure $(\mathrm{J} / \mathrm{kg} \cdot \mathrm{K})$

$D \quad$ pool diameter $(\mathrm{cm})$

$E \quad$ effective emissive power of flame at the surface

E $\left(\mathrm{W} / \mathrm{m}^{2}\right)$

$E_{f} \quad$ emissive power of flame at the surface $\left(\mathrm{W} / \mathrm{m}^{2}\right)$

$F_{12}$ geometric view factor between flame and object

$F_{12, H}$ geometric view factor from a cylinder to a horizontal

$F_{12, H}$ object

$g \quad$ acceleration of gravity $\left(\mathrm{m} / \mathrm{s}^{2}\right)$

$H \quad$ flame height (m)

$\dot{m} \quad$ mass loss rate or burning rate $(\mathrm{g} / \mathrm{s})$

$\dot{q}^{\prime \prime} \quad$ radiative heat flux $\left(\mathrm{W} / \mathrm{m}^{2}\right)$

$\dot{q}^{\prime \prime}(r)$ radiative heat flux in radial direction $\left(\mathrm{W} / \mathrm{m}^{2}\right)$

$\dot{q}^{\prime \prime}(z)$ radiative heat flux in vertical direction $\left(\mathrm{W} / \mathrm{m}^{2}\right)$

$\dot{Q} \quad$ heat release rate $(\mathrm{kW})$

$\dot{Q}_{r} \quad$ total radiative energy output to surrounding (kW)

$\dot{Q}^{*} \quad$ dimensionless heat release rate

$r \quad$ radial distance from the centerline(m)

$R \quad$ radius of vortex core (m)
$V_{\theta} \quad$ tangential velocity $(\mathrm{m} / \mathrm{s})$

$V^{*}$ dimensionless vertical centerline velocity

$z \quad$ vertical distance from the ground (m)

$Z \quad$ normalized vertical distance

Greek

$\alpha \quad$ entrainment coefficient

$\Gamma \quad$ circulation $\left(\mathrm{m}^{2} / \mathrm{s}\right)$

$\bar{\Gamma}$ average circulation along the height $\left(\mathrm{m}^{2} / \mathrm{s}\right)$

$\Gamma^{*}$ dimensionless circulation

$\varepsilon_{f} \quad$ effective emissivity

$\rho_{\infty}$ density of ambient air $\left(\mathrm{kg} / \mathrm{m}^{3}\right)$

$\tau \quad$ atmospheric transmissivity to thermal radiation

$\chi_{r} \quad$ radiative heat loss fraction

$\chi_{f b, r}$ radiative heat feedback fraction

\section{superscripts}

per time

" per area 
$R_{0} \quad$ radial distance between the centerline and vertical radiative heat flux sensors $(\mathrm{m})$

$T$ temperature (K)

$T_{\infty} \quad$ temperature of ambient air (K)

$\Delta T_{z} \quad$ temperature difference between centerline and ambient (K)

$V_{z} \quad$ vertical centerline velocity $(\mathrm{m} / \mathrm{s})$

\section{subscripts}

$\begin{array}{ll}\infty & \text { ambient air } \\ r & \text { radial direction } \\ z & \text { vertical direction }\end{array}$

\section{INTRODUCTION}

Fire whirls, generally caused by an unexpected vortex flow effect on the flame burning, are often reported in forest and urban fires with devastating damage to life and property, and also contribute to spot fires by picking up large firebrands and scattering them over a wide area [1-3]. During the past half century, a number of theoretical and experimental studies have been conducted to provide a basic understanding of the fire whirl phenomenon. By analytical means, hydrodynamic equations were usually simplified and applied to seek the fluid mechanical properties of fire whirl (e.g. Byram and Martin [4], Morton [5], and Muraszew et al. [6]), but generally without considering its combustion characteristics. Morton [5] especially examined the origin, generation and amplification of concentrated vorticity of fire whirl by analysis of relevant vorticity equations. The burning behaviors including burning rate, flame height and flame temperature were mainly investigated by experimental researchers, such as Emmons and Ying [7] by rotating screen-flame model, Soma and Saito [2] and Kuwana et al. [3] utilizing bench-scale model by scaling laws, and Lei et al. [8] by fixed frame-flame model. In recent years, numerical simulations [9,10] have also been widely used for solving the Navier-Stokes or vorticity equations of fire whirl, in order to interpret the detailed fluid flow structure. However, considerable simplified sub-models were generally utilized to describe the chemical and physical processes for numerical modeling.

Until now there is obviously shortage of knowledge upon the initiation mechanism, vertical velocity and radiative heat transfer of fire whirl. In view of this fact, this work conducted experiments of fire whirl by a medium-scale facility for analysis. The drag force on the root of fire whirl flame is indicated to be closely correlated to the initiation and decay processes of fire whirl. The experimental data for vertical centerline velocity and radiative heat transfer of fire whirl are subject to theoretical analysis in details, and some correlations are established.

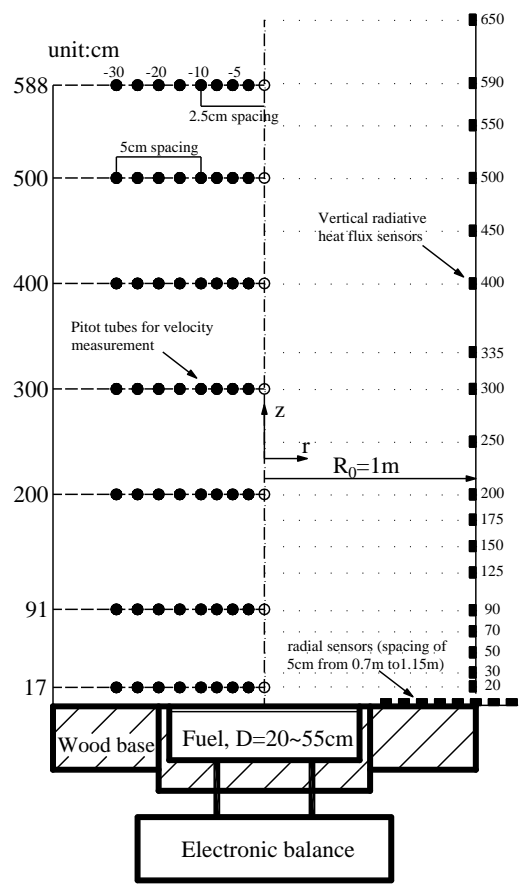

Fig. 1. Schematic for fire whirl experiments. 


\section{EXPERIMENTAL}

The medium-scale fire whirl facility was a square enclosure made of tempered glasses, with dimensions of $2 \mathrm{~m} \times 2 \mathrm{~m} \times 15 \mathrm{~m}$. The channel was open at the top. Each wall had a uniform $20 \mathrm{~cm}$ wide vertical gap at its corner so that the entrained air induced by the burning flame could enter into the channel, thus imparting a rotational flow necessary for fire whirl formation. The base table $(2 \mathrm{~m} \times 2 \mathrm{~m})$ was made of pine wood with a round hole $(60 \mathrm{~cm}$ in diameter and $10 \mathrm{~cm}$ in depth) in the center. Different sizes of pools, with diameter of $20-55 \mathrm{~cm}$ and depth of $10 \mathrm{~cm}$, were placed into the hole for the experiments, with n-heptane $(97 \%)$ as the fuel. A water layer was used to keep the initial surface height constant in all tests.

As shown in Fig. 1, the velocities in both vertical and tangential directions were measured by calibrated temperature-compensated bi-directional pitot tubes (series 160S pitot tubes produced by Dwyer Instruments, Inc.) and Type-K (chromel-alumel) thermocouples of $0.4 \mathrm{~mm}$ in diameter. Radiative heat flow meters (TS-30 produced by Captec Entreprise) were used to measure the radiative heat fluxes in the vertical and radial directions, and the fuel mass versus time was recorded by electronic balance with a precision of $0.1 \mathrm{~g}$. In addition, video was applied to monitor the flame height. The whole facility was placed in a large test hall and all the doors and windows were closed during tests.

\section{RESULTS AND DISCUSSION}

\section{Burning Rate of Fire Whirl: Drag Force Effect}

Before ignition, fuel mass loss was just due to the fuel vaporization at normal room temperature. When the fuel was ignited, it initially burned like a general pool fire. Then the air entrained from the tangential direction began to interact with the upward buoyant plume, making the flame start to incline with a gradually-increased rotation around the vertical axis. Obviously an upward drag force was generated on the fuel or pool surface. Therefore, the mass loss rate in this stage was the sum of the burning rate and the fuel release rate by the drag force. Within a very short period, fire whirl formed, as identified by the phenomenon of that the flame suddenly stood straight up and then revolved around the central axis. After that, the drag force was considered to be constant with small fluctuation, which supported the quasi-steady burning. Therefore, the mass loss rate was just the fire whirl burning rate in this stage. In the last stage of extinction (nearly without burning), the mass loss rate was observed to be positive, which was considered to be due to the loss of drag force.

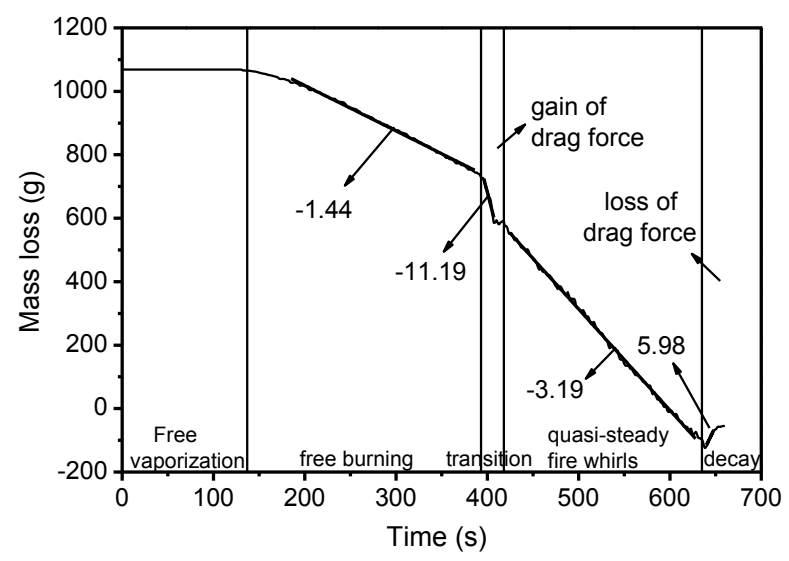

Fig. 2. Mass loss versus time in fire whirl experiment $(D=25 \mathrm{~cm})$.

The above observation and judgment can be verified by the data of mass loss. Figure 2 shows the mass loss versus time for fire whirl with a pool of $25 \mathrm{~cm}$ in diameter. Obviously, the whole process can be definitely divided into five stages, i.e. the fuel vaporization, free burning, transition to the fire whirl, quasi-steady burning, and fire whirl decay. Linear regressions of the data show that the mass loss rate was $-1.44 \mathrm{~g} / \mathrm{s}$ in the free burning stage, $-11.19 \mathrm{~g} / \mathrm{s}$ in the transition stage, $-3.19 \mathrm{~g} / \mathrm{s}$ in the quasi-steady burning stage, and finally $5.98 \mathrm{~g} / \mathrm{s}$ in the fire whirl decay stage. In the transition stage, the rate of drag force with time was $8.00-9.65 \mathrm{~g} / \mathrm{s}$, and the drag force was evaluated to be $96.00-115.80 \mathrm{~g}$, i.e. $0.94-1.13 \mathrm{~N}$. 
A 15-point regression was utilized to obtain mass loss rate versus time (Fig. 3), which shows a large fluctuation without regularity on the moments of gaining or losing the drag force, and the mass loss rate in the stage of quasi-steady burning fluctuates with a specified amplitude and cycle, more significant than that of the free burning.

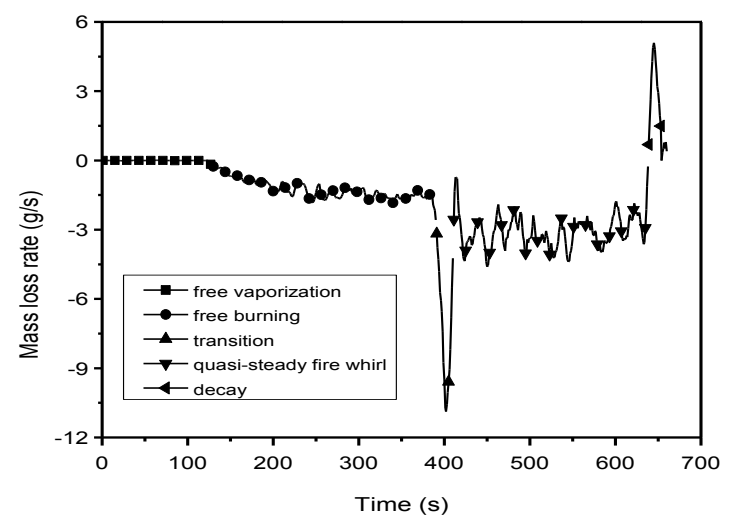

Fig. 3. Mass loss rate versus time in fire whirl experiment $(D=25 \mathrm{~cm})$.

The variation of mass loss rate with time as described above can be clearly explained by mass transfer mechanism. As we know, mass transfer can be caused by diffusion and convection of fuel gases. Fuel vaporization is only controlled by steady diffusion, and free burning is mainly controlled by buoyancy which induces non-steady convection. In the stage of quasi-steady burning, both buoyancy and drag force control the burning, inducing higher instability with strong convection. In this sense, it can be implied that the flow instabilities, as evidenced by the flame wander, may be caused by the drag force which suppresses turbulent fluctuations [8].

\section{Vertical Centerline Velocity of Fire Whirl}

Figure 4 shows the data of $2 \mathrm{~s}$ averaged pressure difference and temperature versus time, for fire whirl with pool diameter of $50 \mathrm{~cm}$. The radiation errors involved in the temperature data used in this work were not corrected. As indicated, within tens of seconds the pressure differences increased sharply, and then remained quasi-steady until extinction. In comparison, more time was needed for the temperature to achieve quasi-steady state. This is true especially for larger value of $z$ and $r$. Here the ideal gas assumption and Bernoulli equation were used in the velocity calculations.

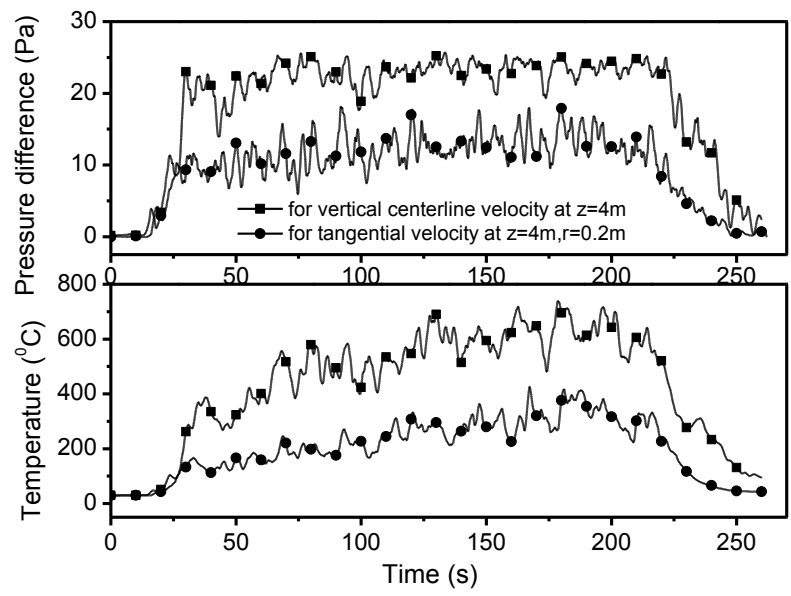

Fig. 4. Data of pressure difference and temperature versus time at $z=4 \mathrm{~m}$ and $r=0$ and $20 \mathrm{~cm}$ respectively $(D=50 \mathrm{~cm})$. 
In tests it was observed that the fire whirl flame spiraled fast and steadily around its central axis. This is considered to be controlled by the balance between the pressure difference force and the centrifugal force. The central core of fire whirl held high temperature and low pressure. Referring to the vertical momentum equation, the vertical velocity is induced by buoyancy due to the density difference between the central core and the ambience. The density difference in fire whirl is obviously caused by the coupled effects of the temperature and pressure difference. In comparison, the density difference in general pool fires is just due to the temperature difference.

McCaffrey [11] suggested that the centerline velocity of a general pool fire satisfies $V_{z} / \sqrt{2 g z \Delta T_{z} / T_{\infty}}=$ constant. However, this correlation is invalid for fire whirls. In this work, the values range from 0.57 to 2.17 . This obviously indicates that the pressure difference characterized by circulation has a significant effect on the centerline velocity of fire whirl.

In order to extract the correlation of the centerline velocity of fire whirl, all the factors which affect the velocity should be first distinguished. Basically, the buoyant plume and concentrated vortex should be included in correlation analysis. We recall the ideal plume velocity correlation [12]

$V_{z}=\left(\frac{25}{48 \alpha^{2}} \cdot \frac{\dot{Q} g}{\pi c_{p, \infty} T_{\infty} \rho_{\infty}}\right)^{1 / 3} \cdot z^{-1 / 3}$

where $\alpha$ is a dimensionless entrainment coefficient. The vortex can be characterized by two parameters, i.e. the radius $R$ of the vortex core in solid-body rotation and the circulation $\Gamma$, which appeared in Kuwana's analysis for the flame height of fire whirl [3]. In our test, the circulation $\Gamma=2 \pi r V_{\theta}$ is nearly constant independent of $r$ out of flame. Therefore, the fire whirl centerline velocity can be expressed by

$V_{z}=f\left(\dot{Q}, z, g, c_{p}, T_{\infty}, \rho_{\infty}, R, \Gamma\right)$

Applying the $\pi$ theorem of dimensionless analysis to the above equation leads to

$V_{z}^{*}=\phi\left(\dot{Q}^{*}, \Gamma^{*}, R / z\right)$

where $V_{z}^{*}=V_{z} / \sqrt{g z}$ is the dimensionless velocity, i.e. Froude number, representing the ratio of inertial force to gravity force; $\dot{Q}^{*}=\dot{Q} /\left(c_{p} \rho_{\infty} T_{\infty} z^{5 / 2} g^{1 / 2}\right)$, the dimensionless heat release rate and $\Gamma^{*}=\Gamma / \sqrt{g z^{3}}$, the dimensionless circulation, respectively reflect the effect of excess temperature and pressure difference on the centerline velocity; $R / z$ is considered to be constant by assuming that the core radius increases linearly with the height.

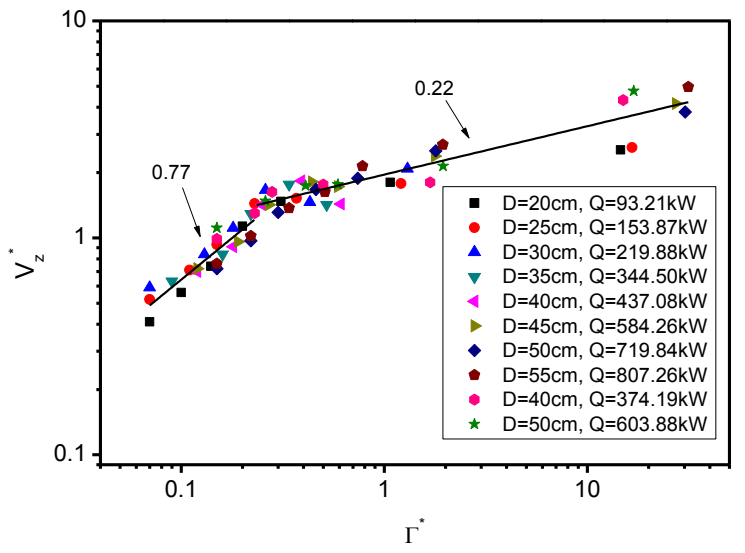

Fig. 5. Dimensionless vertical centerline velocity versus dimensionless circulation. 
By the above consideration, the data of $V_{z}^{*} \sim \Gamma$ were plotted in Fig. 5, which shows that the dimensionless velocity first increases fast and then slowly, with the following correlation

$$
\begin{cases}V_{z}^{*} \sim \Gamma^{* 0.22} & 0.01 \leq \dot{Q}^{*} \leq 46.1 \\ V_{z}^{*} \sim \Gamma^{* 0.77} & 0.002 \leq \dot{Q}^{*} \leq 0.01\end{cases}
$$

By observations and image analysis, it is interesting to note that most of the data points with $0.01 \leq \dot{Q}^{*} \leq 46.1$ fall into the flaming zone, and most of the points with $0.002 \leq \dot{Q}^{*} \leq 0.01$ are in the plume zone. In the form of $Z=z /\left[D^{-0.815}\left(\dot{Q} \cdot \bar{\Gamma}^{2}\right)^{0.33}\right]$ where $\bar{\Gamma}$ is the average circulation, the two zones could be rewritten as $0.003 \leq Z \leq 0.090$ and $0.085 \leq Z \leq 0.166$, respectively corresponding to the flaming zone $Z \leq 0.11$ and plume zone $Z \geq 0.11$ suggested by Lei et al. [8]. Thus, the fire whirl centerline velocity increases versus the height by the relation of $V_{z} \sim z^{0.17} \Gamma^{0.22}$ in the flaming zone, and decreases versus the height by the relation of $V_{z} \sim z^{-0.66} \Gamma^{0.77}$ in the plume zone. Note this is not obvious in the narrow transition stage from flaming zone to plume zone.

\section{Radiation Distribution of Fire Whirl}

Figures 6 and 7 are respectively the 2 s-averaged radiative heat flux versus time at $r=1 \mathrm{~m}, z=0.7 \mathrm{~m}$ and $r=0.7 \mathrm{~m}, z=0$. As observed, long periods are required to achieve steady heat fluxes.

\section{Theoretical Model}

Recall that two common semi-empirical models, i.e. the point source model and the solid flame model, are generally used to evaluate the radiative heat flux from a pool fire to external objects. The point source model assumes that all of the radiation energy from the fire is emitted from a single point, whereas the solid flame model considers the flame to be a solid surface involving a simple shape like cone or cylinder, with constant emissive power. The fire whirl flame could be well regarded as a cylindrical and blackbody homogeneous emitter, according to its shape and the analysis above. The diameter and height of the cylinder are respectively the pool diameter $D$ and flame height $H$ which can be determined from flame video or from the flame height correlation [8]. Thus, the emitted energy from the fire whirl can be expressed as

$\chi_{r} \dot{Q}=\pi D H E_{f}$

where $\chi_{r}$ is the radiative fraction; $\dot{Q}$ is the total heat release rate; $E_{f}$ is the emissive power of the flame at the surface. The radiative heat flux $\dot{q}^{\prime \prime}$, from the flame to a nearby object is

$\dot{q}^{\prime \prime}=\tau E F_{12}$

where $E$ is the effective emissive power of the flame, the product of emissive power and effective emissivity $\varepsilon_{f} ; \tau$ is the atmospheric transmissivity to thermal radiation; $F_{12}$ the geometric view factor between the object and the flame, which is solely a function of the object location, the flame height and diameter. The widely used formula for $F_{12}$ from a heat cylinder to a horizontal object is reproduced below [13].

$F_{12, H}=\frac{(B-1 / S)}{\pi \sqrt{B^{2}-1}} \tan ^{-1} \sqrt{\frac{(B+1)(S-1)}{(B-1)(S+1)}}-\frac{(A-1 / S)}{\pi \sqrt{A^{2}-1}} \tan ^{-1} \sqrt{\frac{(A+1)(S-1)}{(A-1)(S+1)}}$

where $S=2 r / D, h=2 H / D, A=\left(h^{2}+S^{2}+1\right) / 2 S, B=\left(1+S^{2}\right) / 2 S$. 


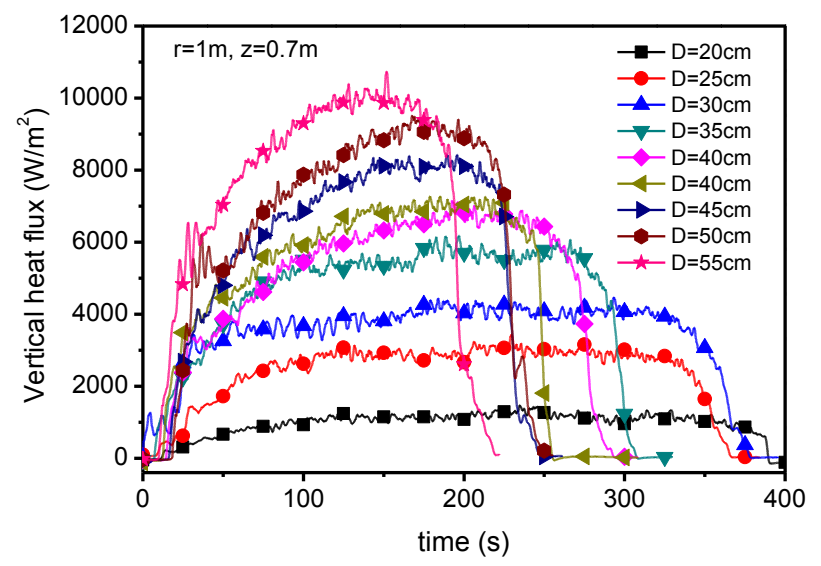

Fig. 6. Vertical radiative heat fluxes versus time.

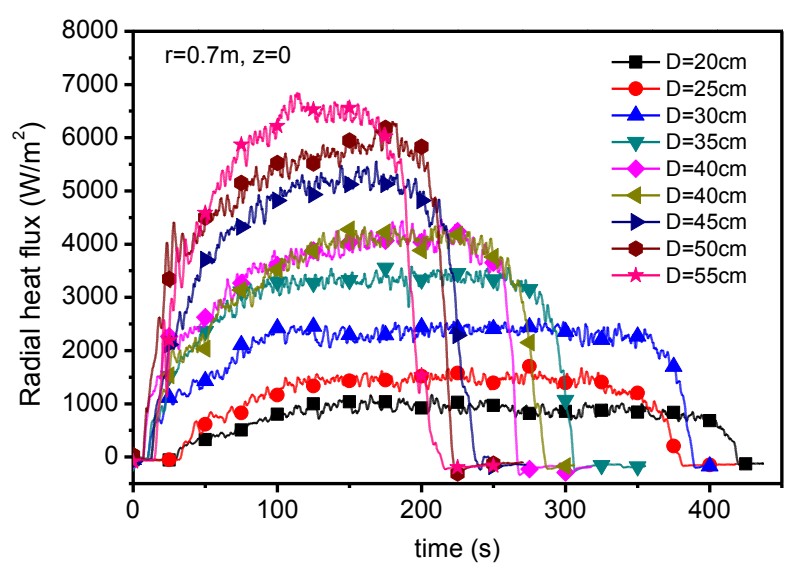

Fig. 7. Radial radiative heat flux versus time.

Incorporating Eq. 5 to Eq. 6 results in

$\dot{q}^{\prime \prime}=\frac{\tau \varepsilon_{f} \chi_{r}}{\pi} \cdot F_{12} \cdot \frac{\dot{Q}}{D H}$

The effective emissivity increases with the pool diameter, while the radiation fraction decreases with the pool diameter, and the transmissivity is independent of the pool diameter. Thus, the assumption of constant dimensionless parameters $\left(\tau \varepsilon_{f} \chi_{r}\right) / \pi$ would be reasonably used in the following analysis.

\section{Radiative Heat Flux Distribution}

According to Eq. 8, the radiative heat flux $\dot{q}^{\prime \prime}$ is the function of the heat release rate $\dot{Q}$, pool diameter $D$, flame height and position $(r, z)$. Since $r=R_{0}$, the vertical radiative heat flux $\dot{q}^{\prime \prime}(z)$ can be written as

$$
\dot{q}^{\prime \prime}(z)=f\left(\dot{Q}, D, H, R_{0}, z\right)
$$

Scaling analysis leads to

$$
\dot{q}^{\prime \prime}(z) H R_{0} / \dot{Q}=\varphi\left(z / H, R_{0} / H, R_{0} / D\right)
$$


Assume that the ratio of $R_{0}$ to $H$ or that of $R_{0}$ to $D$ has a weak influence on the dimensionless heat flux, the relation $\dot{q}^{\prime \prime}(z) H R_{0} / \dot{Q} \sim z / H$ can be employed to fit the radiative heat flux distribution in the vertical direction. By the same method with $z=0$, the relation $\dot{q}^{\prime \prime}(r) H^{2} / \dot{Q} \sim r / H$ could also be derived for the radial radiative heat flux distribution. The fire whirl radiative heat flux distributions thus calculated are shown in Fig. 8 and Fig. 9.

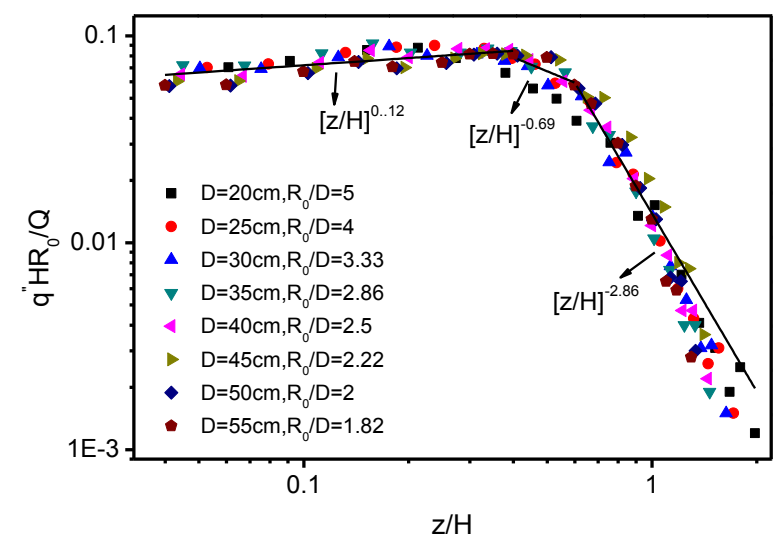

Fig. 8. Radiative heat flux distribution in the vertical direction.

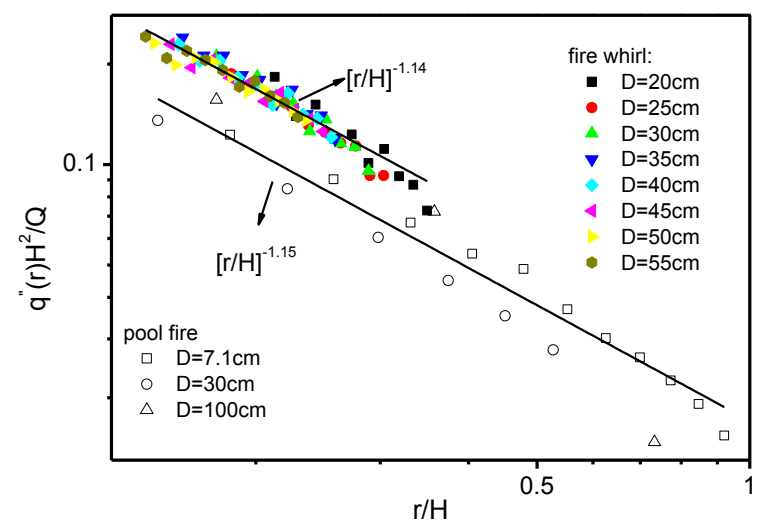

Fig. 9. Radiative heat flux distribution in the radial direction (pool fire data [14]).

As shown, the non-dimensional vertical radiative heat flux correlates well with the vertical distance normalized by the flame height for different pool diameters, with different ratios of $R_{0}$ to $D$. The dimensionless vertical radiative heat flux firstly increases and then decreases slowly, and finally decays rapidly versus the normalized distance. This is considered to be due to the variation of the emissive power in vertical direction. The maximum flux appears at a relatively lower vertical position in all scales, i.e. $z / H \approx 0.4$, while recall that the corresponding value is nearly 0.5 for general pool fires $[14,15]$.

It is found that both fire whirls and general pool fires fit the relation of $\dot{q}^{\prime \prime}(r) H^{2} / \dot{Q} \sim r / H$, with nearly the same exponents, as shown in Fig. 9. For the fire whirl, the correlation is expressed by

$\dot{q}^{\prime \prime}(r)=0.027\left(\frac{r}{H}\right)^{-1.14} \cdot \frac{\dot{Q}}{H^{2}}$

where the radial distance $r$ remains within $0.7-1.15 \mathrm{~m}$ for different pool diameters, or $r / H$ ranges within 0.14-0.35. 
In order to expand the application scope of Eq. 11, comparison of Eq. 8 and Eq. 11 results in $F_{12, H} \sim(r / H)^{-1.14} D / H$, and then Eq. 7 is utilized for verification, with $r$ ranging from half of the diameter to infinite (five times of the flame height), rather than the experimental measurement range. The results shown in Fig. 10 indicate that the Eq. 7 and Eq. 8 could fit any radial position, and the application scope of Eq. 11 could be expanded as $r>D / 2$. In addition, it is reasonable to assume the combination of parameters $\tau \varepsilon_{f} \chi_{r} / \pi$ to be constant, although with several scatters as seen in Fig. 10.

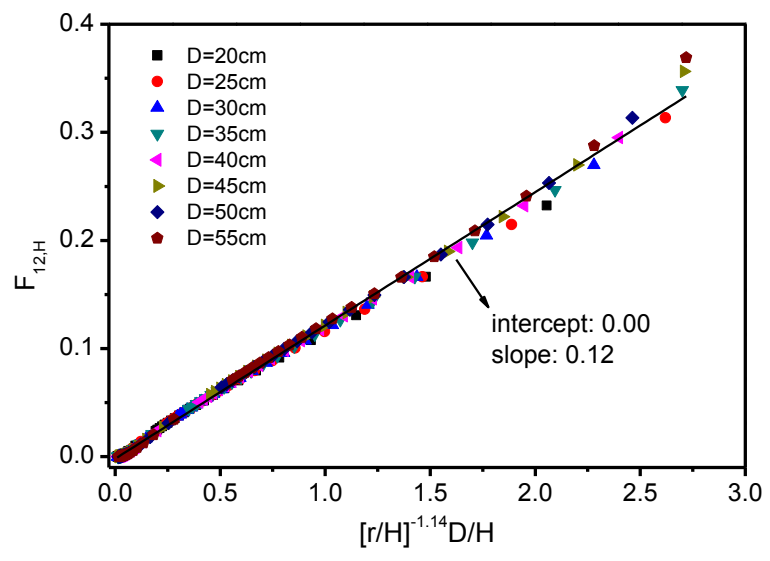

Fig. 10. Geometric view factor in the radial direction.

\section{Total Radiative Energy Output and Radiative Fraction}

The vertical and radial flux distributions are numerically integrated to obtain the total radiative energy output of the fire whirl plume, by using the expression

$$
\dot{Q}_{r}=2 \pi\left(\int r \cdot \dot{q}^{\prime \prime}(r) \cdot d r+R_{0} \int \dot{q}^{\prime \prime}(z) \cdot d z\right)
$$

The radiative fraction can then be calculated by the formula

$$
\chi_{\mathrm{r}}=\dot{Q}_{\mathrm{r}} / \dot{Q}
$$

In our calculation, Eq. 11 was used in the range of $r>D / 2$ to $0.7 \mathrm{~m}$ where no experimental data were available due to the protection of the heat flux sensors. The total radiative energy output and radiative fraction are given in Table 1 for different pool diameters. The radiative fraction is nearly constant with the mean value of $42 \%$. In comparison, the radiative fraction for heptane pool fires with pool diameters ranging from $4.6 \mathrm{~cm}$ to $100 \mathrm{~cm}$ was reported to be $30 \%$ [14].

\section{Radiative Heat Feedback}

The heat transfer from the flame to the fuel surface and the fuel releases from the pool constitute a positive loop. The two processes reach a dynamical balance for the steady burning state of fire whirl, as characterized by the fact that the heat feedback per unit time equals the heat flux needed to vaporize the fuel. The heat could be fed back to fuel surface by conductive, convective and radiative heat transfer processes. It is worth mentioning that the variation of radiative heat flux along the pool surface was essentially flat for the luminous fires, as proved by Hamins et al. who used the experimental results of the $30 \mathrm{~cm}$ heptane and toluene pool fires [16]. In order to assess the relative contribution of the radiative heat feedback process for the fire whirl, an assumption is made that the radiative heat flux variation with the radial distance is constant in the range of $r=0$ to $D / 2$, which equals the radiative heat flux at $r=D / 2$ calculated by Eq. 11 . The radiative heat feedback fraction $\chi_{f b, r}$, i.e. the ratio of the radiative heat feedback to the total heat feedback, is shown in Table 1. It is obvious that the radiative heat feedback is 
underestimated to some extent, since the radial radiative heat flux decreases slowly with the radial distance. However, the results imply that the radiative heat feedback fraction in fire whirls increases with the pool diameter, similarly as general pool fires.

Table 1. Experimental data of this work.

\begin{tabular}{ccccccc}
\hline$D(\mathrm{~cm})$ & $\dot{m}(\mathrm{~g} / \mathrm{s})$ & $H(\mathrm{~m})$ & $\dot{Q}(\mathrm{~kW})$ & $\dot{Q}_{r}(\mathrm{~kW})$ & $\chi_{r}$ & $\chi_{f b, r}$ \\
20 & 2.09 & 3.29 & 93.21 & 38.38 & $41 \%$ & $39 \%$ \\
25 & 3.45 & 3.79 & 153.87 & 67.28 & $44 \%$ & $42 \%$ \\
30 & 4.93 & 3.98 & 219.88 & 91.84 & $42 \%$ & $47 \%$ \\
35 & 7.50 & 4.44 & 334.50 & 142.25 & $43 \%$ & $49 \%$ \\
40 & 9.80 & 4.50 & 437.08 & 183.4 & $42 \%$ & $55 \%$ \\
45 & 13.10 & 4.62 & 584.26 & 257.99 & $44 \%$ & $59 \%$ \\
50 & 16.14 & 4.87 & 719.84 & 294.19 & $41 \%$ & $62 \%$ \\
55 & 18.10 & 5.01 & 807.26 & 322.44 & $40 \%$ & $66 \%$ \\
\hline
\end{tabular}

\section{CONCLUSIONS}

In this work, the initiation mechanism, vertical velocity and radiation heat transfer of fire whirls were investigated using a medium-scale facility and theoretical calculations. The data for burning rate versus time revealed that a drag force has significant effects on the initiation and decay of a fire whirl. Two important factors, i.e. excess temperature and the pressure difference between the central zone and ambient, were proposed to establish the correlation of the vertical centerline velocity for fire whirls. The radiation from a fire whirl was also analyzed by examining the radiative heat flux spatial distribution, total radiative energy output, radiative fraction and radiant heat feedback. The major conclusions are summarized as follows:

(1) The drag force plays an important role in the initiation and decay of a fire whirl.

(2) The swirl-induced pressure difference, described by circulation, plays an important role in the vertical centerline velocity which increases as a function of height by the correlation $V_{z} \sim z^{0.17} \Gamma^{0.22}$ in the flaming zone, and $V_{z} \sim z^{-0.66} \Gamma^{0.77}$ in the plume zone.

(3) The vertical radiative heat flux firstly increases and then decreases slowly, and finally collapses with the vertical distance normalized by the flame height, with a maximum value at $z / H \approx 0.4$ for different pool diameters. The variation of radiative heat flux in the radial direction can be correlated by $\dot{q}^{\prime \prime}(r) \sim r^{-m}$ with the exponent being approximately 1.1 for both heptane fire whirls and general heptane pool fires.

(4) Fire whirls exhibit a higher burning rate and radiative fraction as compared to general pool fires. Additionally, the radiative heat feedback fraction of fire whirls increases with pool diameter, similarly as general pool fires.

\section{ACKNOWLEDGEMENT}

This work was sponsored by National Natural Science Foundation of China under Grants 51076148 and R\&D Special Fund for Public Welfare Industry (forestry, 200704027). Naian Liu was supported by the Fok Ying Tung Education Foundation. Koyu Satoh was supported by Chinese Academy of Sciences Visiting Professorship for Senior International Scientists (Grant No. 2009z2-31). Correspondence to this paper should be addressed to Prof. Naian LIU, liunai@ustc.edu.cn.

\section{REFERENCES}

[1] Graham, H.E., (2003) Fire Whirlwind Formation as Favored by Topography and Upper Winds, Fire Management Today 63: 59-62. 
[2] Soma, S. and Saito, K., (1991) Reconstruction of fire whirls using scale models, Combustion and Flame 86: 269-284, http://dx.doi.org/10.1016/0010-2180(91)90107-M

[3] Kuwana, K., Sekimoto, K., Saito, K., and Williams, F.A., (2008) Scaling fire whirls, Fire Safety Journal 43: 252-257, http://dx.doi.org/10.1016/j.firesaf.2007.10.006

[4] Byram, G. and Martin, R., (1970) The modeling of fire whirlwinds, Forest Science 16: 386-399.

[5] Morton, B., (1970) The Physics of Fire Whirl, Fire Research Abstract and Reviews 8: 1-19.

[6] Muraszew, A., Fedele, J.B., and Kuby, W.C., (1979) The fire whirl phenomenon, Combustion and Flame 34: 29-45, http://dx.doi.org/10.1016/0010-2180(79)90077-4

[7] Emmons, H.W. and Ying, S.-J., "The fire whirl," Symposium (International) on Combustion, Proceedings of the Combustion Institute, 1967, 11(1): 475-488, http://dx.doi.org/10.1016/s0082-0784(67)80172-3

[8] Lei, J., Liu, N., Zhang, L., Chen, H., Shu, L., Chen, P., Deng, Z., Zhu, J., Satoh, K., and de Ris, J.L., "Experimental research on combustion dynamics of medium-scale fire whirl," Symposium (International) on Combustion, Proceedings of the Combustion Institute, 33(2): 2407-241, http://dx.doi.org/10.1016/j.proci.2010.06.009

[9] Battaglia, F., Rehm, R.G., and Baum, H.R., (2000) The fluid mechanics of fire whirls: An inviscid model, Physics of Fluids 12: 2859-2867, http://dx.doi.org/10.1063/1.1308510

[10] Zhou, R. and Wu, Z.N., (2007) Fire whirls due to surrounding flame sources and the influence of the rotation speed on the flame height, Journal of Fluid Mechanics 583: 313-345, http://dx.doi.org/10.1017/S0022112007006337

[11] McCaffrey, B.J., "Purely Buoyant Diffusion Flames: Some Experimental Results," National Bureau of Standards Report NBSIR 79-1910, Washington, D.C., 1979, 52 p.

[12] Karlsson, B. and Quintiere, J.G., Enclosure Fire Dynamics, CRC Press LLC, New York, 2000, $72 \mathrm{p}$.

[13] Shokri, M. and Beyler, C.L., (1989) Radiation from Large Pool Fires, Journal of Fire Protection Engineering 1: 141-149, http://10.1177/104239158900100404

[14] Klassen, M.E. and Gore, J.P., "Structure and Radiation Properties of Pool Fires," National Institute of Standards and Technology Report NIST GCR 94-651, Gaithersburg, MD, 1994, 153 p.

[15] Hamins, A., Kashiwagi, T., and Buch, R.R., "Characteristics of Pool Fire Burning," American Society for Testing and Materials Report ASTM STP1284, Indianapolis, IN, 1996, 30 p.

[16] Hamins, A., Fischer, S.J., Kashiwagi, T., Klassen, M.E., and Gore, J.P., (1994) Heat Feedback to the Fuel Surface in Pool Fires, Combustion Science and Technology 97: 37-62, http://dx.doi.org/10.1080/00102209408935367 